\title{
Une doctrine implicite de la déterritorialisation
}

ou les nouveaux espaces de la puissance et de la ressource selon la Présidence française

\section{Frédéric Giraut}

\section{OpenEdition}

Journals

Édition électronique

URL : http://journals.openedition.org/echogeo/11553

DOI : 10.4000/echogeo. 11553

ISSN : 1963-1197

Éditeur

Pôle de recherche pour l'organisation et la diffusion de l'information géographique (CNRS UMR 8586)

Référence électronique

Frédéric Giraut, «Une doctrine implicite de la déterritorialisation », EchoGéo [En ligne], Sur le Vif, mis en ligne le 17 décembre 2009, consulté le 01 mai 2019. URL : http://journals.openedition.org/ echogeo/11553; DOI : 10.4000/echogeo.11553

Ce document a été généré automatiquement le 1 mai 2019.

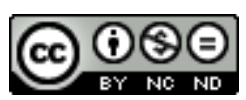

EchoGéo est mis à disposition selon les termes de la licence Creative Commons Attribution - Pas d'Utilisation Commerciale - Pas de Modification 4.0 International 


\section{Une doctrine implicite de la déterritorialisation}

ou les nouveaux espaces de la puissance et de la ressource selon la Présidence française

\section{Frédéric Giraut}

1 «La Russie a le droit de défendre l'intérêt des russophones en dehors de ses frontières » «Il faut cesser de raisonner en termes de zones et de territoires, mais en termes de gens qui y vivent ». Quels rapports entre ces proclamations apparemment frappées au coin du bon sens, mais susceptibles de faire exploser tous les principes de relations internationales pour la première et ceux d'aménagement du territoire redistributif pour la seconde?

Elles émanent du même homme, Nicolas Sarkozy président de la République française qui fut temporairement en charge de la diplomatie européenne comme Président de l'Union, et auparavant longtemps candidat à la Présidence française et ministre de l'intérieur et de l'aménagement des... territoires, justement. Le parallèle pourrait s'arrêter là tant la problématique des relations internationales semble éloignée de celle de l'aménagement des territoires nationaux, régionaux et locaux. En fait, ces différents principes énoncés bien sûr séparément, constituent des éléments disparates d'une véritable doctrine cohérente de la déterritorialisation, au profit d'une organisation spatiale en bastions ou espaces de la puissance associés à des zones d'influence et d'exploitation.

On retrouve là une combinaison d'éléments interprétable par la théorie de la gouvernementalité de Michel Foucault appliquée aux territoires. Gouvernementalité qui dépasse le stade de la souveraineté et qui est basée sur la "sécurité ", comprise chez Foucault au sens d'une mise en ordre spatiale qui assure la discipline, organise et contrôle le mouvement et permet l'exploitation. Pour rendre à la doctrine implicite de la déterritorialisation de la Présidence française sa complexité et sa richesse, nous proposons d'enrichir la grille de lecture foucaldienne par des emprunts aux théories économiques du «Public choice» et de la «Nouvelle économie géographique » qui constituent pour cette doctrine des références là encore implicites. 
4 L'homme politique Sarkozy est un être hyperterritorial: longtemps député-maire, président de conseil général et ministre de l'intérieur, il a conçu sa carrière politique à partir des cadres territoriaux les plus stables et les plus anciens de l'édifice territorial français, se tenant toujours éloigné des cadres plus fluctuants et nouveaux que sont l'intercommunalité et la région. Par ailleurs, malgré les proclamations rappelées plus haut, il supporte en tant que Président de la République, une nouvelle politique des banlieues, propose de rénover l'architecture territoriale française et promeut l'idée d'un Grand Paris. Il est par ailleurs un fervent défenseur de l'idée de fondements identitaires de la nation bien gardée par un ministère et par des frontières étanches à la migration non choisie. De même, il est le garant d'une orthodoxie continentale pour la définition de l'Europe dans laquelle « l'Asie Mineure » n'aurait pas sa place. Enfin, il aurait finalement obtenu en 2008 le retrait de l'armée russe sur ses frontières linguistiques entérinant au passage l'annexion de deux régions géorgiennes. Hyperterritorial donc !

5 A y regarder de plus prêt, ces différents engagements territoriaux dans le cadre national ou international, ne sont pas contradictoires avec les professions de foi initialement rappelées, ils les confirmeraient même.

6 Notons tout d'abord la focalisation sur les aspects sécuritaires, y compris dans le Plan banlieue qui a notamment introduit des « unités territoriales de quartier ». L'idée de zone prioritaire bénéficiant de la solidarité nationale est essentiellement réduite à sa forme Zone franche, tandis que les Zones urbaines sensibles deviennent le cadre d'une contractualisation directe dite « d'autonomie » avec certains de ses résidents. Ces zones ne sont pas vraiment des territoires dira-t-on, et tout l'enjeu est de les associer dans une gestion métropolitaine et sociale plus large plutôt que de les stigmatiser ou les isoler, mais le choix est fait de l'extraction des élites des Zones urbaines sensibles d'une part et $\mathrm{du}$ cantonnement policier de l'autre. Les nouvelles opportunités pour en sortir individuellement par les mesures de promotion sociale et scolaire individualisées et au mérite incarnent l'espoir, non pas d'une transformation et d'une intégration des quartiers, mais d'une récompense personnelle pour y avoir subi et vaincu la marginalisation. Le recours cosmétique à la notion de "busing" (expérience américaine de fermeture des établissements scolaires les plus frappés par la relégation sociale, et distribution des élèves dans les établissements plus attractifs de l'agglomération) irait dans le sens de l'option de la dissolution des structures ghéttoïsées et donc de la reconstruction d'un territoire urbain plus intégré. Mais seul le mot est retenu pour déboucher sur des mesures expérimentales non associées à la fermeture des établissements ghetto. Avec également l'entretien et la reproduction de la ressource humaine sur site par les zones franches, destinées il est vrai à favoriser l'émergence d'un entrepreneuriat, on est donc très loin d'une politique d'intégration territoriale, mais plutôt dans une logique de contrôle et de reproduction combinée à celle de l'extraction des maigres ressources périphériques que constituent les élites des quartiers sensibles.

7 Le chantier de l'instauration d'un Grand Paris parait, quant à lui, aller au contraire dans le sens d'une nouvelle territorialité métropolitaine intégrée. Il est ainsi le symbole de la transfiguration d'un Président ex-maire de Neuilly et ex-président du Conseil général des Hauts-de-Seine (soit les collectivités territoriales les plus riches de France dans leurs catégories respectives), jadis campé sur un certain égoïsme territorial (en termes de répartition du logement social d'une part et des revenus des collectivités territoriales aux échelles nationale et régionale d'autre part) et aujourd'hui converti à l'intégration à l'échelle de toute l'agglomération. D'autant plus surprenant que des élus locaux 
franciliens ${ }^{2}$ proches du Président se sont longtemps illustrés par différentes initiatives parlementaires pour mettre en cause les fondements de l'intercommunalité et pour créer des clubs de communes nanties pouvant bénéficier des subsides d'Etat réservés à la promotion de la coopération intercommunale. Ils ont en fait promu en France la doctrine du "Public Choice". Elle postule les effets bénéfiques de la concurrence entre les gouvernements locaux au sein des agglomérations. Elle est fondée aux Etats-Unis sur le postulat de mobilités résidentielles et productives qui permettent aux acteurs de "voter avec leurs pieds", c'est à dire de déménager vers les collectivités locales qui mènent les politiques qui leur conviennent le mieux en termes de production et de gestion de biens publics. Cette doctrine était étrangère jusque là au débat public français et même européen, si ce n'est au Royaume Uni de Margaret Thatcher lorsque le Grand Londres fut démantelé de même que les comtés métropolitains. En effet, en Europe où la mobilité intra urbaine des résidents et des entreprises est limitée par la nature même de la ville et son épaisseur historique, les élus s'opposent sur les questions de solidarité, de redistribution, de fiscalité et de planification, mais conviennent généralement de l'intérêt des économies d'échelle et de la coopération appliquée à la gestion des services. Mais que les tenants du «Public Choice» se rassurent, le projet de Grand Paris s'oriente vers la création d'une grande opération d'équipement régional ${ }^{3}$ et de développement métropolitain basé sur le principe d'isolats d'innovation et de production pour les «Clusters » et d'isolat directionnel pour la Défense, tous déconnectés du tissu urbain ${ }^{4}$. Le projet a pour effet à court terme de priver la région d'une part et la ville-centre d'autre part de leurs prestiges politiques et donc de leurs statuts de potentiel tremplin politique national, et d'organiser la concurrence à plusieurs niveaux en interne sans attribution de capacité planificatrice. La manière dont l'actuel Conseil général des Hauts-de-Seine récupère sur l'île Seguin un grand projet culturel de capitale et de ville globale pour en faire un élément de son propre dispositif d'offre départementale de loisirs de luxe en dit déjà long sur les pratiques à venir dans un Grand Paris nouvel espace équipé de la dynamique métropolitaine édifié sur les décombres d'un district central et cosmopolite de ville globale et d'une région métropolitaine en capacité de planification.

L'actuelle réforme de l'architecture territoriale française, une fois l'arbitrage présidentiel rendu, propose une série d'innovations. Celles-ci loin de simplifier le système ne suppriment en fait que certaines expériences originales pour assurer au final la promotion de nouveaux espaces pseudo-métropolitains à partir des villes secondaires étendues à leurs arrière-pays ou mises en réseau. Exit les pays ruraux et leurs conseils de développement, ces territoires émergents et expérimentaux de projet qui incarnaient la mise en place de véritables scènes de définition de projets de territoire partagés issus d'un processus participatif. Pour le projet, la priorité est donnée aux seules structures métropolitaines dont le monde rural et ses villes petites et moyennes ne constitueraient plus qu'un arrière pays. La référence aux pays ne disparaît pas pour autant, elle reste plébiscitée par le marketing territorial qu'il s'agisse de la promotion de pays touristiques, de terroirs labellisés ou de la dénomination de nombre de communautés de communes qui se sont découvertes du pays de quelque chose dans une nouvelle toponymie qui évite ainsi d'arbitrer entre les lieux fusionnés. Tendance à l'utilisation non contrôlée d'un label décidément bien pratique pour tout territoire intercommunal en quête de légitimité, les pays qui se portent donc bien dans leur version marketing politique vont continuer à s'afficher comme étendard de nombreux et hétérogènes territoires ruraux de promotion économique. Au final, les circonscriptions électorales des nouveaux conseillers territoriaux, les nouveaux cantons, prévus par la même réforme risquent de capter la 
notion en lieu et place des périmètres innovants de projet et de coopération où s'élaborait en lien avec les régions et parfois l'Europe une dynamique à géométrie variable de développement ouverte sur la société civile.

9 Le passage du Président Sarkozy aux questions internationales, pourtant d'une toute autre nature, s'est effectué à partir de cette culture et de ce qui apparait comme une doctrine implicite de la déterritorialisation: défense et affirmation des cadres de la puissance et organisation de leur périphérie en tant que lieux de la ressource et de l'échange inégal. Cela apparaît clairement dans le positionnement initial sur le conflit géorgien et le droit d'intervention de la puissance russe au nom des russophones. Positionnement traduit dans l'accord initial de cessez-le-feu avec la Russie. L'incroyable omission de la notion d'intégrité territoriale se combinait alors avec l'acceptation du principe de "mesures additionnelles de sécurité" sans référence à la localisation de telles mesures. Les déclarations ultérieures faites sur l'Ukraine à l'occasion du lancement d'un accord de libre échange sur sa vocation et son caractère européen constituent également des concessions symboliques aux impérialismes de la puissance et de l'identité. En effet, le recours récurrent à une définition essentialiste d'un continent culturel plutôt que le seul soutien au choix démocratique d'une nation souveraine ne disqualifie finalement pas sur le fond, et malgré le rappel à cette occasion du caractère non négociable de l'intégrité territoriale ukrainienne, la tentation d'un dépeçage de ces confins ukrainiens entre une partie proprement européenne et une partie russe, toutes deux pouvant apparaître comme des glacis périphériques légitimes.

Le projet d'Union méditerranéenne également cher, fut un temps, à la Présidence française est une autre manifestation non moins importante de l'influence de cette doctrine spatiale, forgée dans le microcosme politique français, et appliquée aux questions internationales. Même s'il reste flou dans ses modalités, ce projet, inventé d'abord pour sortir de l'impasse que constituait la promesse d'exclusion définitive de la Turquie de l'Union européenne, pourrait se traduire, à la suite du Partenariat Euroméditerranéen, par la proposition suivante faite aux Etats d'Afrique du Nord et du Proche-Orient: voulez-vous profiter de notre puissance dans la proximité ? Accordez nous alors un partenariat privilégié pour l'exploitation sélective de votre marché et de vos ressources au sens large, naturelles mais aussi humaines. ${ }^{5}$. On voit là l'influence des théories de la nouvelle économie géographique une fois appliquée aux suds avec les contraintes déterministes et dépendantistes engendrées par les rendements croissants (effets d'agglomération) qui offrent un avantage définitif aux centres constitués et le path dependency (l'enchaînement historique) qui confère également un avantage aux situations acquises. Les périphéries n'ont donc qu'à attendre des espaces de la puissance (ici l'Europe) que de la condescendance et les retombées généreuses d'un partenariat déséquilibré. On est en fait bien loin de la réalité et des aspirations des pays émergents et on touche les limites d'une vision spatiale, certes cohérente à toutes les échelles, mais profondément décalée par rapport aux besoins d'intégration et de régulation.

11 La vision proposée, qui se fait doctrine implicite, est celle d'un monde qui reste quadrillé avec des frontières étanches pour les questions sécuritaires, mais déterritorialisé et à la souveraineté sélective pour les questions politiques et économiques ${ }^{6}$. Ceci au profit des bastions de la puissance qui cantonnent les périphéries dans une fonction d'espaces sécurisés exploitables. 


\section{BIBLIOGRAPHIE}

ANTHEAUME B. \& GIRAUT F. (eds.), 2005, Le territoire est mort, Vive les territoires! , Paris: IRD Editions.

BLETON-RUGET A., BODINEAU P. \& SYLVESTRE J.-P. (dirs.), 2002, "Pays" et territoires. De Vidal de la Blache aux lois d'aménagement et de développement du territoire, Dijon : Ed. Universitaires de Dijon.

BUCHANAN, J. 1965. «An economic theory of clubs», Economica, novembre, 1-14.

CHANET J.-F., 2001,“Terroirs et pays : mort et transfiguration ?”, Vingtième Siècle. Revue d'histoire 69, 61-81.

DARBOT-TRUPIANO S., 2007, « Le Partenariat euro-méditerranéen : une tentative d'intégration maladroite », L'Espace Politique [En ligne], 2 | 2007-2, http://espacepolitique.revues.org/ index844.html

DEAN M., 1999, Governmentality : Power and rule in Modern Society, New York : Sage.

ELDEN S., 2005, "Missing the point: globalization, deterritorialization and the space of the world", Transactions of the Institute of British Geographers 30, 8-19.

ELDEN S., 2007, “Governmentality, calculation, territory”, Environment and Planning D: Society and Space 25, 562-580.

ESTÈBE P., 2008, Gouverner la ville mobile, Paris : PUF.

Espace Géographique, 2007, « Nouvelle économie géographique et géographie : quel dialogue ? ", Espace géographique 2007/3, Tome 36, 193-214.

FOUCAULT M., 1975, Surveiller et punir. Naissance de la prison. Paris : Gallimard.

FOUCAULT M., 2004, Sécurité, territoire, population : Cours au Collège de France (1977-1978), Paris : Ed M. Sennellart (Gallimard).

GERBAUX F. (ed.), 1999, Utopie pour le territoire : cohérence ou complexité ?, La Tour d'Aigues : Ed. de l'Aube.

GIRAUT F., 2009, «Les ambiguïtés de la nouvelle doctrine spatiale de la Banque mondiale”, Cybergéo (Revue Européenne en ligne de Géographie), Débats, 8 p. http://www.cybergeo.eu/ index22695.html

GIRAUT F. \& LAJARGE R., 1999, "Que vont les Pays devenir ? Entre normalisation, cacophonie et abandon : cinq scénarii" in "Pays" et développement local, NOROIS, 46(181), 219-222.

GIRAUT F. \& VANIER M., 1999, "Plaidoyer pour la complexité territoriale" in Utopie pour le territoire : cohérence ou complexité ?, F. Gerbaux (ed.), La Tour d'Aigues : Ed. de l'Aube, 143-172.

GRATALOUP C., 2009, L'invention des continents, Paris : Larousse.

LASCOUMES P., 2007, "La Gouvernementalité: de la critique de l'État aux technologies du pouvoir", Le Portique 13-14 (Foucault: usages et actualités).

OFFNER J.-M., 2006, “Les territoires de l'action publique locale. Fausses pertinences et jeux d'écarts", Revue française de science politique 56(1), 27-47. 
RAFFESTIN C., 1980, Pour une géographie du pouvoir, Paris : LITEC.

REYNAUD A., 1981, Société, espace et justice, Paris : PUF.

SCHOLZ F., 2005, "The theory of fragmenting development", Geographische Rundchau International Edition 1(2), 4-11

TIEBOUT C., OSTROM V. \&WARREN R., 1961. « The organization of government in metropolitan areas : A theoretical enquiry ", American Political Science Review LV(4),831-842.

VANIER M., 2008, Le pouvoir des territoires: Essai sur l'interterritorialité, Paris : Economica.

\section{NOTES}

1. La citation exacte est : «Il est parfaitement normal que la Russie veuille défendre ses intérêts ainsi que ceux des Russes en Russie et des Russophones à l'extérieur de la Russie" (Nicolas Sarkozy, Moscou, 12 aout 2008).

2. Notamment M. Patrick Beaudouin (député du Val-de-Marne, Maire de Saint-Mandé) et M. Philippe Pemezec, (ex-député des Hauts-de-Seine, Vice-président du Conseil général, Maire du Plessis-Robinson), auteurs du « Livre noir de l'intercommunalité » et de la proposition de loi (n -2741) de décembre 2005 à l'Assemblée nationale. Leurs communes sont par ailleurs, pour la première rétive avec sa voisine Vincennes à une intégration dans la communauté d'agglomération de l'Est parisien, et pour la seconde membre de la très homogène communauté d'agglomération "des Hauts de Bièvre", communauté « défensive et a minima » selon les mots de ses promoteurs.

3. Ce que dénonce dans ses dernières prises de position l'architecte Jean Nouvel qui a été associé aux réflexions initiales et est l'auteur de l'un des projets commandités. Cf Nouvel J., 2009, "Le projet du Grand Paris est menacé de s'enliser dans la confusion". Le Monde du 201009.

4. cf Blanc C., 2009, "L'ambition nationale du Grand Paris", Le Monde, Lundi 23 novembre.

Gili F., 2009, “Grand Paris, l'occasion manquée ?”, Le Monde, Mardi 24 novembre.

Behar D. \& Estebe P., 2009, “Le projet du Grand Paris n'est pas métropolitain”, Le Monde, Lundi 23 novembre.

5. «L'Europe a besoin du dynamisme démographique et des marchés émergents de la rive méridionale ; le Sud a besoin du savoir-faire de l'Europe et d'une Europe solidaire pour affronter le choc de l'ouverture commerciale » in Guigou J.-L., 2007, « Les “quartiers d'orange” Nord-Sud : réguler la mondialisation par les espaces régionaux », Le Monde 20/03/07.

Jean-Louis Guigou, ardent défenseur de l'Union Méditerranéenne, est Délégué général de l'Institut de prospective économique du Monde méditerranéen, Paris, créé par la Présidence Française en 2007.

6. «La mondialisation valorise quoi ? les pôles et les réseaux. Notre organisation s'appuie sur quoi ? des circonscriptions et des frontières. Voilà tout le problème! " (Nicolas Sarkozy, Saint Dizier, 20 octobre 2009) 


\section{RÉSUMÉS}

La présidence française a mis en place un ensemble de nouvelles politiques publiques dans le champ de l'aménagement des territoires (Plan banlieue, Grand Paris, Réforme de l'architecture territoriale...), elle a également pris une série d'initiatives dans le champ des relations internationales (Crise géorgienne, Union pour la Méditerranée..). Ces initiatives intérieures et extérieures s'accompagnent de discours sur le territoire, le développement et la souveraineté. Apparemment disparates, sans liens entre elles, voire contradictoires et mues par un certain pragmatisme, ces prises de positions et ces pratiques peuvent être analysées comme relevant d'une doctrine implicite, mais cohérente, qui fonctionne à différentes échelles, de celle de l'agglomération à celle du monde. Le recours aux théories de la gouvernementalité, du public choice et de la nouvelle économie géographique permettent de décrypter les fondements de cette doctrine implicite de la déterritorialisation: quadrillage avec des frontières étanches pour les questions sécuritaires, et souveraineté sélective pour les questions politiques et économiques. Ceci au profit des bastions de la puissance qui cantonnent les périphéries dans une fonction d'espaces sécurisés exploitables.

Is there a coherent doctrine of the French Presidence on territories, sovereignty and development? Regarding these fields, the French Presidence has performed on the one hand the implementation of a new set of spatial planning and local government public policies and on the other hand a European leading role through initiatives in international relations . Including the related discourses, all these initiatives could be interpreted in terms of governementality as being driven by a common and coherent doctrine of deterritorialization which is linked by some aspects with the "New geographic economy" and the "Public choice" theories. The implicit doctrine promote control and closed boundaries in the field of security and migrations but selective sovereignty for economic purpose to the profit of the power strongholds which assign peripheries to a function of exploitable and secured spaces.

\section{INDEX}

Mots-clés : Deterritorialisation, Souveraineté; Centre-periphérie; Présidence française; Gouvernementalité; Public choice ; Nouvelle économie géographique

Keywords : Deterritorialization; Public choice; New economic geography; Governmentality; Center-periphery; French presidence; Sovereignty

\section{AUTEUR}

\section{FRÉDÉRIC GIRAUT}

Frédéric Giraut (Frederic.Giraut@unige.ch) est professeur en géographie politique et aménagement à l'Université de Genève. Il a publié récemment : Boujrouf S., Antheaume B., Giraut F. \& Landel P.-A., (eds.), 2009, Les territoires à l'épreuve des normes: référents et innovations, Contributions croisées sud-africaines, françaises et marocaines, Marrakech et Grenoble: 
Université Cadi Ayyad et Revue Montagnes Méditerranéennes, 526 p. ; Giraut F. \& VacchianiMarcuzzo C., 2009, Territories and Urbanisation in South Africa, Atlas and geo-historical information system (DYSTURB), Paris : IRD Editions. http://www.cartographie.ird.fr/ dysturb.html; Giraut F. \& Houssay-Holzschuch M. (eds.), 2008, Au nom des territoires ! Dossier sur la toponymie, Espace géographique 2. http://www.cairn.info/revue-espace-

geographique-2008-2.htm 